\title{
1 RNAlater and flash freezing storage 2 methods nonrandomly influence 3 observed gene expression in RNAseq 4 experiments
}

Courtney N. Passow ${ }^{1+}$, Thomas J. Y. Kono ${ }^{2+}$, Bethany A. Stahl ${ }^{3}$, James B. Jaggard ${ }^{3}$, Alex C. Keene ${ }^{3}$, Suzanne E. McGaugh ${ }^{*}$

${ }^{1}$ Ecology, Evolution, and Behavior, 140 Gortner Lab, 1479 Gortner Ave, University of Minnesota, Saint Paul, MN 55108

${ }^{2}$ Minnesota Supercomputing Institute, 117 Pleasant Street SE, University of Minnesota, Minneapolis, MN 55455

${ }^{3}$ Department of Biological Sciences, Florida Atlantic University, 5353 Parkside Drive, Jupiter, FL 33458

*Corresponding Author: smcgaugh@umn.edu + Authors contributed equally 


\section{Abstract:}

33 RNA-sequencing is a popular next-generation sequencing technique for assaying

34 genome-wide gene expression profiles. Nonetheless, it is susceptible to biases that are

35 introduced by sample handling prior gene expression measurements. Two of the most

36 common methods for preserving samples in both field-based and laboratory conditions

37 are submersion in RNAlater and flash freezing in liquid nitrogen. Flash freezing in liquid

38 nitrogen can be impractical, particularly for field collections. RNAlater is a solution for

39 stabilizing tissue for longer-term storage as it rapidly permeates tissue to protect cellular

40 RNA. In this study, we assessed genome-wide expression patterns in 30 day old fry

41 collected from the same brood at the same time point that were flash-frozen in liquid

42 nitrogen and stored at $-80^{\circ} \mathrm{C}$ or submerged and stored in RNAlater at room

43 temperature, simulating conditions of fieldwork. We show that sample storage is a

44 significant factor influencing observed differential gene expression. In particular, genes

45 with elevated GC content exhibit higher observed expression levels in liquid nitrogen

46 flash-freezing relative to RNAlater-storage. Further, genes with higher expression in

47 RNAlater relative to liquid nitrogen experience disproportionate enrichment for

48 functional categories, many of which are involved in RNA processing. This suggests

49 that RNAlater may elicit a physiological response that has the potential to bias biological

50 interpretations of expression studies. The biases introduced to observed gene

51 expression arising from mimicking many field-based studies are substantial and should

52 not be ignored.

Keywords: Liquid nitrogen, RNAlater, gene expression, gene length, GC proportion,

55 technical variation

56

57

58

59

60

61

62 


\section{Introduction}

65

66

67

68

69

70

71

72

73

74

75

76

77

78

79

80

81

82

83

84

85

86

87

88

89

90

91

92

93

94

95

96

97

98

99

100

101

102

103

104

105

106

107

108

109

High throughput sequencing technologies, such as RNA-sequencing methods, have revolutionized the quantification of genome-wide expression patterns across a broad range of fields in biological sciences (López-Maury et al. 2008; Wang et al. 2009). However, storage and RNA extraction methods prior to RNA-seq library preparation exert substantial impacts on biological studies, and often account for the majority of variation in a dataset if conditions and protocols are not identical across all samples (Todd et al. 2016). With the rise of RNAlater (Ambion, Invitrogen) as a popular storage method in field-based studies (De Smet et al. 2017; Wille et al. 2018), it is important to quantify if there are systematic biases in gene expression when samples are preserved in RNAlater versus flash-frozen in liquid nitrogen. In our literature review, however, we could find few direct comparisons of RNAseq data obtained from the most common field-preservation method RNAlater and the "gold standard" of flash freezing samples in liquid nitrogen (Alvarez et al. 2015; Wolf 2013) (but see(Cheviron et al. 2011; Choi et al. 2016)). Further, no studies examined whether a systematic bias due to gene characteristics exists for samples preserved in RNAlater.

Currently, two of the most common methods for RNA preservation and storage are flash freezing in liquid nitrogen and preservation in aqueous sulfate salt solutions, such as commercially available RNAlater. Flash freezing, usually through the use of immersing the sample in dry ice or liquid nitrogen, is the most preferred means of stabilizing tissue samples for downstream analysis (Wolf 2013). While preferred, it can often be difficult to access and transport dry ice or liquid nitrogen, particularly in field conditions (Mutter et al. 2004). Hence, in the past decade, it has become common practice, especially in field environments, to store RNAseq-destined samples in RNAlater, a stabilizing solution that minimizes the need to readily process samples or chill the tissue. RNAlater can rapidly permeate tissue to stabilize and protect RNA (Chowdary et al. 2006; Florell et al. 2001). Likewise, RNAlater-immersed samples can be stored safely at room temperature for a week, and longer when stored at colder temperatures. Though, common practice is to store samples in RNAlater in field conditions for much longer than a week. While the exact ingredients of commercial RNAlater are unknown, the Material Safety Data Sheet lists inorganic salt as the major component and the homemade versions contain ammonium sulfate, sodium citrate, ethylenediaminetetraacetic acid (EDTA), and adjustment of $\mathrm{pH}$ using sulfuric acid.

In this study, we quantified the effects of storage condition on gene expression and examined differentially expressed genes for specific characteristics to assay for systematic bias. Individual, Mexican tetra fry (Astyanax mexicanus), were collected from the same brood and stored immediately in liquid nitrogen $(\mathrm{N}=6)$ or RNAlater $(\mathrm{N}=5)$. We specifically asked (1) Does storage condition affect patterns of differential gene expression and if so, (2) Are these effects on gene expression non-random, such that genes with certain features are differentially affected by storage condition? We found that a majority of the variation in gene expression was explained by storage condition. Likewise, we found that genes with higher GC content exhibited higher expression values in liquid nitrogen than RNAlater. Based on these findings, RNAlater-storage may potentially bias biological conclusions of RNAseq experiments. 


\section{Methods}

\section{Sample Collection}

112 Samples for the transcriptome analyses were collected from a surface population of

113 Astyanax mexicanus (total of 8 parents) that had been reared in the Keene laboratory at

114 Florida Atlantic University for multiple generations. Parental fish were derived from wild-

115 caught Río Choy stocks originally collected by William Jeffery. To minimize variation

116 outside of storage methods, all individuals were collected from the same clutch

117 (fertilized on 2016-12-08). Fish were raised in standard conditions, three days prior to

118 experiment, fish were transferred into dishes with 12-21 fish per dish in a 14:10 light-

119 dark cycle. Individuals were raised for 30 days after fertilization under standard

120 conditions, when five individuals were sampled and stored in RNAlater and six

121 individuals were flash frozen in liquid nitrogen and stored at -80 . These fish were a part

122 of a larger experiment and so for 24 hours prior to sampling, fish were kept in total

123 darkness and sampled at 16:00h (10pm). To mimic field conditions, RNAlater

124 individuals were stored at room temperature for 17 days (Camacho-Sanchez et al.

125 2013; Kono et al. 2016). Procedures for all experiments performed were approved by

126 the Institutional Animal Care and Use Committee at Florida Atlantic University (Protocol

127 \#A15-32).

128 RNA extraction, library preparation and sequencing

129 For RNA isolation, all individuals were processed within a week of each other (between

130 2017-01-19 and 2017-01-24), and RNAlater stored individuals were processed 17 days after initial storage (2017-01-24) (Table S1) with the same researcher performing all extractions. Whole organisms ( $<30 \mathrm{mg}$ of tissue) were homogenized using Fisherbrand pellet pestles and cordless motor (Fisher Scientific) in the lysate buffer RLT plus. Total RNA was extracted using the Qiagen RNAeasy Plus Mini Kit (Qiagen) and quantified using NanoDrop Spectrophotometer (Thermo Fisher Scientific), Ribogreen (Thermo Fisher Scientific), and Bioanalyzer (Agilent) to obtain RNA integrity numbers (RIN). All cDNA libraries were constructed at the University of Minnesota Genomics Center on the same day in the same batch. In brief, a total of $400 \mathrm{ng}$ of RNA was used to isolated mRNA via oligo-dT purification. dsDNA was constructed from the mRNA by randomprimed reverse transcription and second-strand cDNA synthesis. Strand-specific cDNA libraries were then constructed using TruSeq Nano Stranded RNA kit (Illumina), following manufacturer protocol. Library quality was assessed using Agilent DNA 1000 kit on a Bioanalyzer (Agilent). To minimize batch effects, barcoded libraries were then pooled and sequenced across multiple lanes of an Illumina HiSeq 2500 to produce 125bp paired-end reads at University of Minnesota Genomics Center (Table S1). All sequence data were deposited in the short read archive (Study Accession ID: RNAlater: SRX3446133, SRX3446136, SRX3446135, SRX3446155, SRX3446156; liquid N2: 


\section{RNAseq quality check}

150 The raw RNA-seq reads were quality checked using Fastqc (Andrews 2014) and 151 trimmed to removed adapters using the program Trimmomatic version 0.33; (Bolger et al. 2014). Trimmed reads were mapped to the Astyanax mexicanus reference genome (version 1.0.2; GenBank Accession Number: GCA_000372685.1; (McGaugh et al. 2014). Mapping was conducted using the splice-aware mapper STAR (Dobin et al. 2013), because it yielded the higher alignment percentage and quality compared to a similar mapping program (HISAT2, results not shown (Kim et al. 2015)). We used Stringtie (version 1.3.3d; (Pertea et al. 2015) (Pertea et al. 2016) to quantify number of reads mapped to each gene in the reference annotation set of the $A$. mexicanus genome, and used the python script provided with Stringtie (prepDE.py) to generate a gene counts matrix (Pertea et al. 2016). R (Team 2014) was used to compare RIN between liquid nitrogen and RNAlater treatments using a nonparametric Kruskal-Wallis test.

Variation in gene expression

To visualize changes in observed gene expression, we performed principal components analysis on a gene counts matrix. Genes with less than 100 counts across all samples were removed from the matrix because genes with low counts bias the differential expression tests (Love et al. 2014). The resulting counts were decomposed into a reduced dimensionality data set with the prcomp() function in $\mathrm{R}$ (Team 2014).

To identify genes that showed the largest difference in observed gene expression between storage conditions, we performed a differential expression analysis between samples flash frozen in liquid nitrogen $(\mathrm{N}=6)$ and samples stored in RNAlater $(\mathrm{N}=5)$ using DESeq2 (Love et al. 2014). DESeq2 normalizes expression counts for each sample and then fits a negative binomial model for counts for each gene. Samples with the same storage condition were treated as replicates, (i.e., the variation due to storage was assumed to be greater than variation among biological samples). This was confirmed in the PCA plot (Figure 1), where PC1 linearly separated samples based on their treatments. P-values for differential expression were adjusted based on the Benjamini-Hochberg algorithm, using a default false discovery rate of at most 0.1 (Love et al. 2014). Genes were labeled as differentially expressed if the Benjamini-Hochberg adjusted P-value was less than 0.1. Log2(RNAlater/liquid nitrogen) values were calculated with DESeq2, and exported for further analysis.

\section{Linear model to determine factors influencing differential expression}

To identify the factors that contribute to the variability in gene expression between preservation methods, we fit a linear model of observed gene expression of all genes as a function of various genomic characteristics. We tested the contributions of mean expression level, annotated gene length, exon number, GC content, presence or absence of simple sequence repeats, and presence or absence of a homopolymer tract to differences in observed gene expression between preservation methods. We used the log2(RNAlater/liquid nitrogen) values from DESeq2 as the measure of change in observed gene expression, and the mean of normalized counts as the mean expression 
193 level. The annotated gene length was calculated as the total length of the gene annotation, including noncoding (i.e., intronic) regions. A simple sequence repeat was defined as two or more nucleotides repeated at least three times in tandem, and a homopolymer tract was defined as a single nucleotide repeated at least six times in tandem in the reference genome. Repeat presence or absence was based only on the reference genome sequence, and were not scored to be polymorphic in the sample. Length and exon number were calculated with a modified version of GTFTools ( $\mathrm{Li}$ 2018). GC content, presence/absence of a simple sequence repeat, and presence/absence of a homopolymer repeat were scored with custom Python scripts available on our GitHub repository. Notably, the reference genome was based off the Pachón cavefish, and it is conceivable that some homopolymers and sequence repeats may not be identical in the surface fish.

We performed model selection on a series of linear models using likelihood ratio tests of nested models. The "full model" was as follows:

$$
Y=0+\beta_{0} M+\beta_{1} G+\beta_{2} L+\beta_{3} E+\beta_{4} S+\beta_{5} H+\beta_{6}(G \times S)+\beta_{7}(G \times H)+\varepsilon,
$$

where $Y$ is $\log 2$ (RNAlater/liquid nitrogen) of expression between treatments, $M$ is the the normalized mean expression value across all samples, $G$ is $G C$ content, $L$ is gene length, $E$ is the total number of exons in the gene, $S$ is SSR presence/absence, and $H$ is homopolymer presence/absence. GC content, gene length, and exon number were treated as continuous variables, and SSR presence and homopolymer presence were treated as categorical variables. Model selection proceeded by testing the contributions of the interaction terms to the variance explained, and removing them if not significant. We tested the terms with the lowest non-significant $t$-values in the regression, and removed them if they did not significantly improve model fit.

Annotation of differentially expressed genes

Since most of the variation was explained by a technical variable (i.e., preservation and storage), we did not expect biologically meaningful annotation. However, we conducted annotation analyses using two different methods. Differentially expressed genes at the 0.05 false discovery rate were converted to homologous zebrafish (Danio rerio) gene IDs for a gene ontology (GO) term enrichment analysis. Duplicate zebrafish gene IDs were removed prior to $\mathrm{GO}$ term enrichment analyses. GO term enrichment was tested with the GOrilla webserver (Eden et al. 2009) (http://cbl-gorilla.cs.technion.ac.il/), with a database current as of 2018-07-07. Other running parameters were left at their default values. In addition, PANTHER analysis (Mi et al. 2016) (http://pantherdb.org/tools/compareToRefList.jsp) was run using 1:1 orthologs between zebrafish and Asytanax with database current as of 2018-04-30. Within the PANTHER suite, we used PANTHER v13.1 overrepresentation tests (i.e., Fisher's exact tests with FDR multiple test correction) with the Reactome v58, PANTHER proteins, GoSLIM, GO, and PANTHER Pathways. For both annotation analyses, they were run with two lists of unranked gene IDs: the target list was the differentially expressed gene IDs (either 
237 higher or lower expression in RNAlater), and the background list was all zebrafish

238 genes genome-wide.

239 Script Availability

240 Scripts to perform all data QC and processing are available at

241 https://github.com/TomJKono/CaveFish_RNAlater

242

243 Results

244 Mapping statistics and annotation

245 RNA sequencing from whole, 30-days post fertilization individuals yielded a total of

$246108,874,500$ reads for individuals stored in liquid nitrogen (mean $=18,145,750 \pm$ stdev

$2471,938,410$ per individual; $N=6$ ) and 82,448,455 reads for individuals stored in RNAlater

248 (mean = 16,489,691 \pm stdev 1,890,519 per individual; $\mathrm{N}=5$ ) (Table 1). While all RIN

249 scores passed the threshold (>7), RIN scores were significantly different between

250 RNAlater and liquid nitrogen treatments (Kruskal-Wallis chi-squared =7.6744, $\mathrm{df}=1, \mathrm{p}$ -

251

252

253

254

255

256 value $=0.005601$; RNAlater mean $\mathrm{RIN}=8.60$, liquid nitrogen mean $\mathrm{RIN}=9.83$ ).

Total yield of reads and number of uniquely mapping reads were not significantly different between treatments $(t=1.4301 ; P=0.1875)$. Samples on average mapped $88.17 \%$ of the reads to the Astyanax mexicanus genome (range: $86.93 \%-89.90 \%$ ), with liquid nitrogen samples mapping on average $88.17 \%$ and RNAlater mapping $87.24 \%$. Filtering of the gene counts matrix to include only genes with $\geq 100$ reads resulted in 15,515 genes being used for both clustering and differential expression analysis. Annotations were extracted from the Astyanax mexicanus annotation file (Astyanax_mexicanus.AstMex102.91.gtf). Distributions of raw and filtered gene expression counts are given in Figure S1.

\section{PCA and Differentially Expressed Genes}

Principal components analysis showed that the major axis of differentiation among the samples was treatment (Figure 1). This corresponds to the first principal component,

265

266 and explains $27.2 \%$ of the variation. Beyond the first principal component, the samples do not cluster into further discernable sub-groups, suggesting that the main axis of differentiation among these samples is their storage conditions (Figure S2 A and B). A total of $2,708(17.5 \%)$ genes were significantly differentially expressed between treatments at the 0.05 significance level (Figure 2). Of these, 1,635 exhibited significantly lower observed expression in RNAlater than liquid nitrogen, and 1,073 exhibited significantly higher observed expression in RNAlater than liquid nitrogen.

\section{Annotation of differentially expressed genes}

273 We expected little GO term enrichment as differences in gene expression would likely 274 be due to differences in preservation techniques, not biological variation. Further, the 275 number of enrichment categories for higher- and lower-expressed genes in RNAlater 
with respect to liquid nitrogen was similar across annotation programs. However, we observed substantially different functional enrichment among genes that were higherand lower-expressed in RNAlater compared to liquid nitrogen across annotation programs.

In the GOrilla analyses, GO term enrichment analysis showed that the genes that were differentially expressed between treatments were spread across a broad range of GO terms. In genes that are significantly lower in RNAlater in comparison to liquid nitrogen, the only significantly enriched GO term is protein autophosphorylation (GO:0046777). In genes that are significantly higher in RNAlater, there were 13 enriched GO terms (after FDR correction; Supplementary Material). These included acyl-CoA, thioester, and sulfur compound metabolic processes, and purine nucleoside, nucleoside, and ribonucleoside bisphosphate metabolic processes. Notable, many of these processes involve replacing the linking oxygen in an ester by a sulfur atom, and if the homemade version of RNAlater is consistent with the commercial recipe, ammonium sulfate is likely the largest component.

The PANTHER suite annotation results were similar to the GOrilla analyses (Supplemental Materials). For genes that were significantly lower in RNAlater compared to liquid nitrogen, very few functional categories were enriched. However, many categories were significantly enriched for genes that were more highly expressed in RNAlater than liquid nitrogen. The most enriched categories in reactome pathways are involved in gene expression and processing of mRNA. Likewise, enriched PANTHER protein classes include RNA binding proteins, mRNA processing and splicing factors, and transcription factors. Enriched GO terms included RNA binding and RNA processing.

This consistent elevation of enrichment of functional categories for genes that are more abundant after an RNAlater treatment suggests that this treatment may be altering the physiology of the tissue.

\section{Genomic Characters Contributing to Differential Expression}

307 We identified four characteristics that contribute significantly to differential gene 308 expression between treatments. Mean expression across samples, GC content, exon number, and homopolymer repeat presence/absence were significant, or nearly significant, terms in the model (Table 2, Figure 3). GC content exhibits the most substantial regression coefficient. The coefficient for GC content is negative, suggesting that genes with higher GC content have a higher relative expression in liquid nitrogen than RNAlater. Mean expression, exon number, and homopolymer repeat presence/absence were significant, or nearly so, such that they exhibited a positive relationship with genes showing higher expression values in RNAlater (i.e., greater mean expression, more exons, having a homopolymer repeat are all related to higher expression in RNAlater). The small regression coefficients of these variable imply, however, that these factors have negligible impacts on differential gene expression observed between preservation methods. 


\section{Discussion}

321 Many sources contribute to variation in observed gene expression. Of these, most researchers are interested in assaying the variation that is due to a biological factor, such as genetic or physiological differences between samples. However, variation due to technical factors, such as noise in hybridization efficacy in microarray studies (Altman 2005) or noise in the number of reads that map to a transcript in RNAseq studies are large sources of variability in observed gene expression, and can substantially influence results (Bryant et al. 2011; Marioni et al. 2008). For RNA-sequencing studies, the sources of technical variation are still being discovered, but can include many aspects of sample handling prior to actual measurement (Mclntyre et al. 2011). Previous microarray studies have compared the two sample handling procedures that were tested in our study, and have found no difference downstream, particularly in differential gene expression patterns (Dekairelle et al. 2007; Mutter et al. 2004). These studies, however, may not apply to the variance profile of RNA-sequencing studies (Romero et al. 2012).

Our results suggest that sample handling is an important factor in variation of observed gene expression. While the total percentages of reads mapped were generally similar between the two treatments, the treatments we tested had a significant impact on RNA quality. Our results suggest that preservation in RNAlater, as opposed to flash freezing, non-randomly impacts gene expression values of over $20 \%$ of the transcriptome, and our results suggest that shorter genes with higher GC content and lower expression are better preserved in liquid nitrogen. Conversely, our results suggest that genes with high GC content or lower mean expression may not be as well preserved with RNAlater (De Wit et al. 2012). The functional enrichment for genes exhibiting significantly higher observed expression in RNAlater than liquid nitrogen indicates that RNAlater may be substantially altering the physiology of the samples during fixation or that RNAlater preserves certain functional categories of genes better than liquid nitrogen. The latter seems more unlikely as it is difficult to hypothesize a mechanism. Further, the converse, does not appear to have extensive enrichment for certain functional categories (i.e., genes that experience presumably worse preservation in RNAlater than liquid nitrogen often do not fall in particular functional categories) .

Based on our results, we recommend that researchers use caution when comparing gene expression values derived from RNAseq datasets that may have variable storage conditions. This is especially important with the growth of genomics technologies and accessibility of public data in repositories such as the NCBI Sequence Read Archive. Many entries in these databases do not routinely report metadata such as storage conditions, posing a serious challenge for data utilization. Further, future work could expand on examination of storage in TRIzol (Fisher Scientific, Hampton, NH) as recent work indicates expression patterns might be substantially different from liquid nitrogen (Kono et al. 2016). Likewise, various taxonomic groups may be more susceptible to variation in storage conditions because they may exhibit different tissue permeability.

Several caveats are important in interpreting our study. While technical variation from storage condition is the dominant contributor to variation in our study, we acknowledge 
that biological variation also contributes to our observations. The samples in each storage condition are separate, whole individuals from the same clutch of fish. Fry at 30 days post fertilization are too small to divide tissues equally into preservation treatments and obtain sufficient RNA quantity for RNAseq. Yet, even if a larger tissue sample was cut and divided, one might expect biological variation due to different cell populations. Additionally, juvenile fish tissue may interact with the RNAlater buffer in different ways from other organisms. However, other studies have demonstrated similar effects between RNAlater and flash freezing. For instance, between preservation methods over 5000 differentially regulated genes have been obtained from Arabidopsis thaliana tissue (c.f. (Kruse et al. 2017)). Though this previous analysis did not assay systematic biases of particular gene attributes to preservation methods, many differentially regulated genes were related to osmotic stress, indicating a strong transcriptional response to RNAlater. Finally, long-term storage temperature is confounded with liquid nitrogen and RNAlater treatments in our study and long-term storage temperature is known to drive RNA integrity (Kono et al. 2016) (Gayral et al. 2013). Our goal was to replicate typical field experiments, where reliable refrigeration is not available for substantial amounts of time, and RNAlater is used as the predominant preservation method. Despite these caveats, our work demonstrates that differing preservation methods and storage conditions non-randomly impact gene expression, which may bias interpretation of results of RNA sequencing experiments. We look forward to future work that more thoroughly quantifies the impact on interpretation of biological signal derived solely from preservation methods.

\section{Acknowledgements}

We thank the University of Minnesota Genomics Center for their guidance and authors acknowledge the Minnesota Supercomputing Institute (MSI) at the University of Minnesota for providing resources that contributed to the research results reported within this paper. URL: http://www.msi.umn.edu. Funding was supported by (1R01GM127872-01 to SEM and ACK). CNP was supported by Grand Challenges in Biology Postdoctoral Program at University of Minnesota College of Biological Sciences. Institutional Animal Care and Use Committee at Florida Atlantic University (Protocol \#A15-32).

\section{Data accessibility}

400 All reads are available in NCBI short read archive under accession numbers SRX3446133, SRX3446136, SRX3446135, SRX3446155, SRX3446156, SRS2736519,

403 SRS2736520, SRS2736523, SRS2736524, SRS2736525, and SRS2736526. Scripts to

404 perform all data handling and analysis tasks are available in a GitHub repository at https://github.com/TomJKono/CaveFish_RNAlater 


\section{References}

406

407

408

409

410

411

412

413

414

415

416

417

418

419

420

421

422

423

424

425

426

427

428

429

430

431

432

433

434

435

436

437

438

439

440

441

442

443

444

445

446

447

448

449

Altman N (2005) Replication, Variation and Normalisation in Microarray Experiments. Applied Bioinformatics 4, 33-44.

Alvarez M, Schrey AW, Richards CL (2015) Ten years of transcriptomics in wild populations: what have we learned about their ecology and evolution? Molecular ecology 24, 710-725.

Andrews S (2014) FastQC: a quality control tool for high throughput sequence data. Version 0.11. 2. Babraham Institute, Cambridge, UK http://www.bioinformatics.babraham.ac.uk/projects/fastqc.

Bolger AM, Lohse M, Usadel B (2014) Trimmomatic: A flexible trimmer for Illumina sequence data. Bioinformatics 30, 2114-2120.

Bryant PA, Smyth GK, Robins-Browne R, Curtis N (2011) Technical variability is greater than biological variability in a microarray experiment but both are outweighed by changes induced by stimulation. PloS one 6, e19556.

Camacho $\square$ Sanchez M, Burraco P, Gomez $\square$ Mestre I, Leonard JA (2013) Preservation of RNA and DNA from mammal samples under field conditions. Molecular Ecology Resources 13, 663-673.

Cheviron ZA, Carling MD, Brumfield RT (2011) Effects of postmortem interval and preservation method on RNA isolated from field-preserved avian tissues. The Condor 113, 483-489.

Choi S, Ray HE, Lai S-H, Alwood JS, Globus RK (2016) Preservation of multiple mammalian tissues to maximize science return from ground based and spaceflight experiments. PloS one 11, e0167391.

Chowdary D, Lathrop J, Skelton J, et al. (2006) Prognostic gene expression signatures can be measured in tissues collected in RNAlater preservative. The journal of molecular diagnostics 8, 31-39.

De Smet L, Hatjina F, loannidis P, et al. (2017) Stress indicator gene expression profiles, colony dynamics and tissue development of honey bees exposed to sublethal doses of imidacloprid in laboratory and field experiments. PloS one 12, e0171529.

De Wit P, Pespeni MH, Ladner JT, et al. (2012) The simple fool's guide to population genomics via RNA $\square$ Seq: an introduction to high $\square$ throughput sequencing data analysis. Molecular Ecology Resources 12, 1058-1067.

Dekairelle A-F, Van der Vorst S, Tombal B, Gala J-L (2007) Preservation of RNA for functional analysis of separated alleles in yeast: comparison of snap-frozen and RNALater $\AA^{\circledR}$ solid tissue storage methods. Clinical Chemical Laboratory Medicine 45, 1283-1287.

Dobin A, Davis CA, Schlesinger F, et al. (2013) STAR: ultrafast universal RNA-seq aligner. Bioinformatics 29, 15-21.

Eden E, Navon R, Steinfeld I, Lipson D, Yakhini Z (2009) GOrilla: a tool for discovery and visualization of enriched $\mathrm{GO}$ terms in ranked gene lists. BMC bioinformatics $10,48$.

Florell SR, Coffin CM, Holden JA, et al. (2001) Preservation of RNA for functional genomic studies: a multidisciplinary tumor bank protocol. Modern pathology 14, 116. 
Gayral P, Melo-Ferreira J, Glémin S, et al. (2013) Reference-free population genomics from next-generation transcriptome data and the vertebrate-invertebrate gap. PLoS genetics 9, e1003457.

Kim D, Langmead B, Salzberg SL (2015) HISAT: a fast spliced aligner with low memory requirements. Nature methods 12, 357.

Kono N, Nakamura H, Ito Y, Tomita M, Arakawa K (2016) Evaluation of the impact of RNA preservation methods of spiders for de novo transcriptome assembly. Molecular Ecology Resources 16, 662-672.

Kruse CP, Basu P, Luesse DR, Wyatt SE (2017) Transcriptome and proteome responses in RNAlater preserved tissue of Arabidopsis thaliana. PloS one 12, e0175943.

Li H (2018) GTFtools: a Python package for analyzing various modes of gene models. bioRxiv.

López-Maury L, Marguerat S, Bähler J (2008) Tuning gene expression to changing environments: from rapid responses to evolutionary adaptation. Nature Reviews Genetics 9, 583.

Love MI, Huber W, Anders S (2014) Moderated estimation of fold change and dispersion for RNA-seq data with DESeq2. Genome biology 15, 550.

Marioni JC, Mason CE, Mane SM, Stephens M, Gilad Y (2008) RNA-seq: an assessment of technical reproducibility and comparison with gene expression arrays. Genome research.

McGaugh SE, Gross JB, Aken B, et al. (2014) The cavefish genome reveals candidate genes for eye loss. Nature communications 5, 5307-5307.

McIntyre LM, Lopiano KK, Morse AM, et al. (2011) RNA-seq: technical variability and sampling. BMC genomics 12, 293.

Mi $\mathrm{H}$, Huang X, Muruganujan A, et al. (2016) PANTHER version 11: expanded annotation data from Gene Ontology and Reactome pathways, and data analysis tool enhancements. Nucleic Acids Research 45, D183-D189.

Mutter GL, Zahrieh D, Liu C, et al. (2004) Comparison of frozen and RNALater solid tissue storage methods for use in RNA expression microarrays. BMC genomics $5,88$.

Pertea M, Kim D, Pertea GM, Leek JT, Salzberg SL (2016) Transcript-level expression analysis of RNA-seq experiments with HISAT, StringTie and Ballgown. Nature protocols 11, 1650 .

Pertea M, Pertea GM, Antonescu CM, et al. (2015) StringTie enables improved reconstruction of a transcriptome from RNA-seq reads. Nature biotechnology 33, 290.

Romero IG, Ruvinsky I, Gilad Y (2012) Comparative studies of gene expression and the evolution of gene regulation. Nature Reviews Genetics 13, 505.

Team RC (2014) R: A language and environment for statistical computing.

. In: R Foundation for Statistical Computing.

Todd EV, Black MA, Gemmell NJ (2016) The power and promise of RNA $\square$ seq in ecology and evolution. Molecular ecology 25, 1224-1241.

Wang Z, Gerstein M, Snyder M (2009) RNA-Seq: a revolutionary tool for transcriptomics. Nature Reviews Genetics 10, 57. 
bioRxiv preprint doi: https://doi.org/101101/379834; this version posted July 29,2018 . The copyright holder for this preprint (which was not certified by peer review) is the author/funder, who has granted bioRxiv a license to display the preprint in perpetuity. It is made available under aCC-BY-NC-ND 4.0 International license.

495 Wille $M$, Yin $\mathrm{H}$, Lundkvist $\AA$, et al. (2018) RNAlater ${ }^{\circledR}$ is a viable storage option for avian 496 influenza sampling in logistically challenging conditions. Journal of virological methods 252, 32-36.

498 Wolf JB (2013) Principles of transcriptome analysis and gene expression quantification:

499 an RNA $\square$ seq tutorial. Molecular Ecology Resources 13, 559-572.

500

501 


\section{Tables}

503 Table 1: Reported are the number of reads (after adapter trimming) used as input for 504 the mapping software (STAR), number of reads that uniquely mapped to the reference 505 genome, and the percent of reads that mapped to the reference genome.

506

\begin{tabular}{lllll}
\hline Sample Name & Treatment & Input reads & $\begin{array}{l}\text { Uniquely mapped } \\
\text { reads }\end{array}$ & $\%$ Mapped \\
\hline CHOY-16-01 & Liquid N2 & $20,162,412$ & $18,125,738$ & $89.90 \%$ \\
CHOY-16-04 & Liquid N2 & $15,760,631$ & $13,812,190$ & $87.64 \%$ \\
CHOY-16-05 & Liquid N2 & $18,025,208$ & $16,015,383$ & $88.85 \%$ \\
CHOY-16-08 & Liquid N2 & $16,368,007$ & $14,584,314$ & $89.10 \%$ \\
CHOY-16-11 & Liquid N2 & $17,997,036$ & $15,126,300$ & $89.61 \%$ \\
CHOY-16-12 & Liquid N2 & $20,561,206$ & $18,221,558$ & $88.62 \%$ \\
CHOY-16-R-01 & RNAlater & $17,984,846$ & $15,643,479$ & $86.98 \%$ \\
CHOY-16-R-03 & RNAlater & $17,064,911$ & $14,913,653$ & $87.39 \%$ \\
CHOY-16-R-04 & RNAlater & $13,585,649$ & $11,809,525$ & $86.93 \%$ \\
\hline CHOY-16-R-05 & RNAlater & $15,692,250$ & $13,716,160$ & $87.41 \%$ \\
CHOY-16-R-2 & RNAlater & $18,120,799$ & $15,851,038$ & $87.47 \%$ \\
\hline & & & & \\
\hline & & & & \\
\hline & & & & \\
\hline
\end{tabular}


510 Table 2: Terms in the linear model that explain differences in expression between

511 RNAlater store and liquid nitrogen freezing and $-80^{\circ} \mathrm{C}$ storage.

\begin{tabular}{lccccc}
\hline \multicolumn{1}{c}{ Term } & Sum Sq & Df & F-value & $\begin{array}{c}\text { Estimate } \\
(\mathrm{SE})\end{array}$ & P-value \\
\hline Mean Expression & 8 & 1 & 3.4682 & $\begin{array}{c}3.893 \mathrm{e}-06 \\
(1.642 \mathrm{e}-06)\end{array}$ & 0.06258 \\
GC Proportion & 76 & 1 & 31.3766 & $\begin{array}{c}-1.092 \\
(0.2837)\end{array}$ & $2.164 \mathrm{e}-08$ \\
& & & & $\begin{array}{c}0.01941 \\
(1.340 \mathrm{e}-03)\end{array}$ & $<2.2 \mathrm{e}-16$ \\
Exon Number & 508 & 1 & 209.9133 & $\begin{array}{c}0.05196 \\
(0.02582)\end{array}$ & 0.04420 \\
HPR Presence & 10 & 1 & 4.0495 & & \\
\hline
\end{tabular}




\section{Figure Legends}

514

515

516

517

518

Figure 1: Principal components analysis plot showing PC1 and PC2 for each sample. RNAlater samples (red) are linearly separated from liquid nitrogen samples (blue) by PC1.

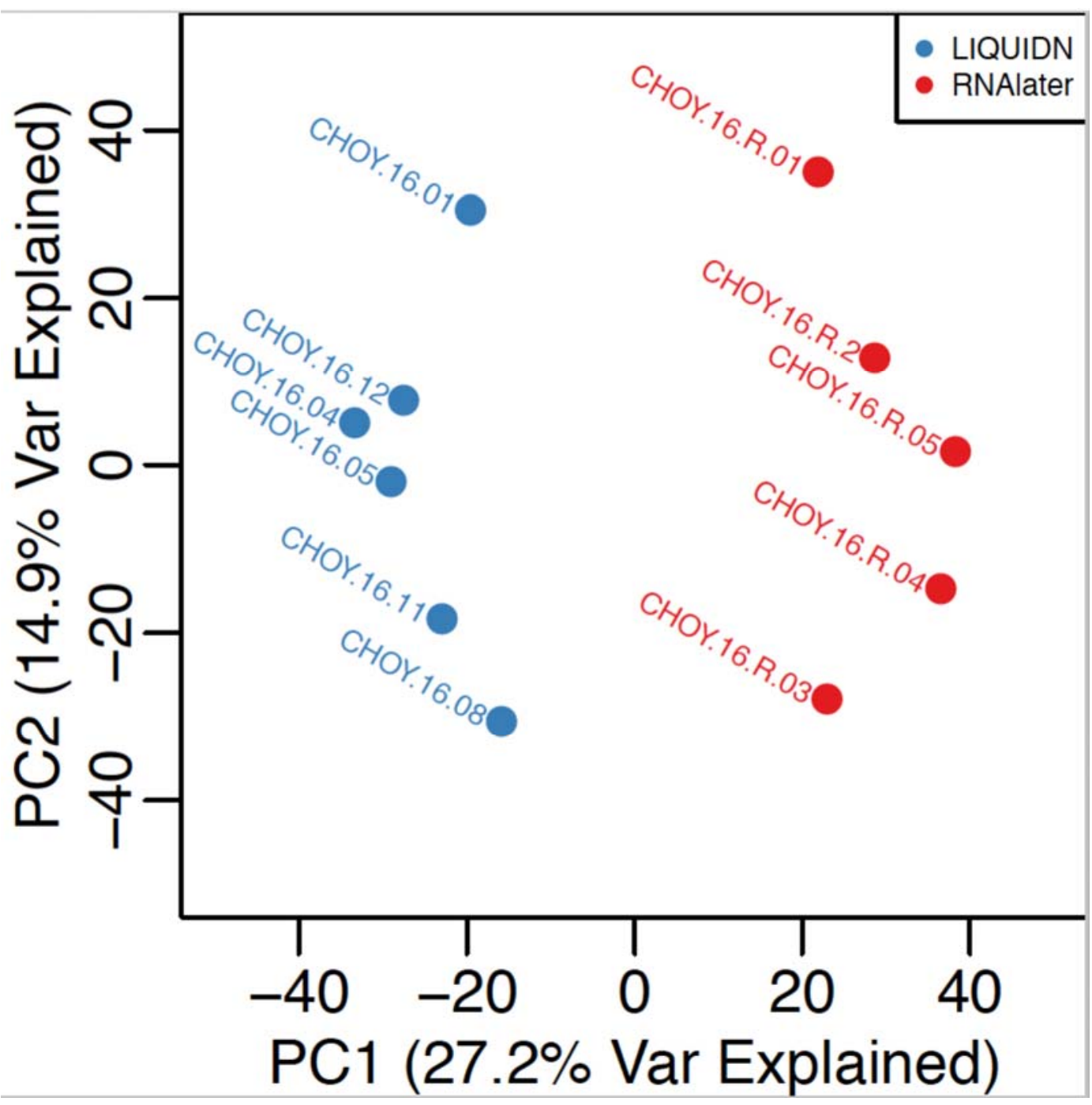


522 Figure 2: Clustering heatmap showing genes that are differentially expressed among RNAlater samples and liquid nitrogen samples. Gene expression values have been normalized by sample, then centred about 0 for each gene. This heatmap contains differentially expressed genes (after FDR correct with $p<0.05$ ) including 1,073 genes that with higher expression values in the RNAlater treatment relative to the liquid nitrogen treatment, and 1,635 genes that exhibited lower expression values the

528 RNAlater treatment.

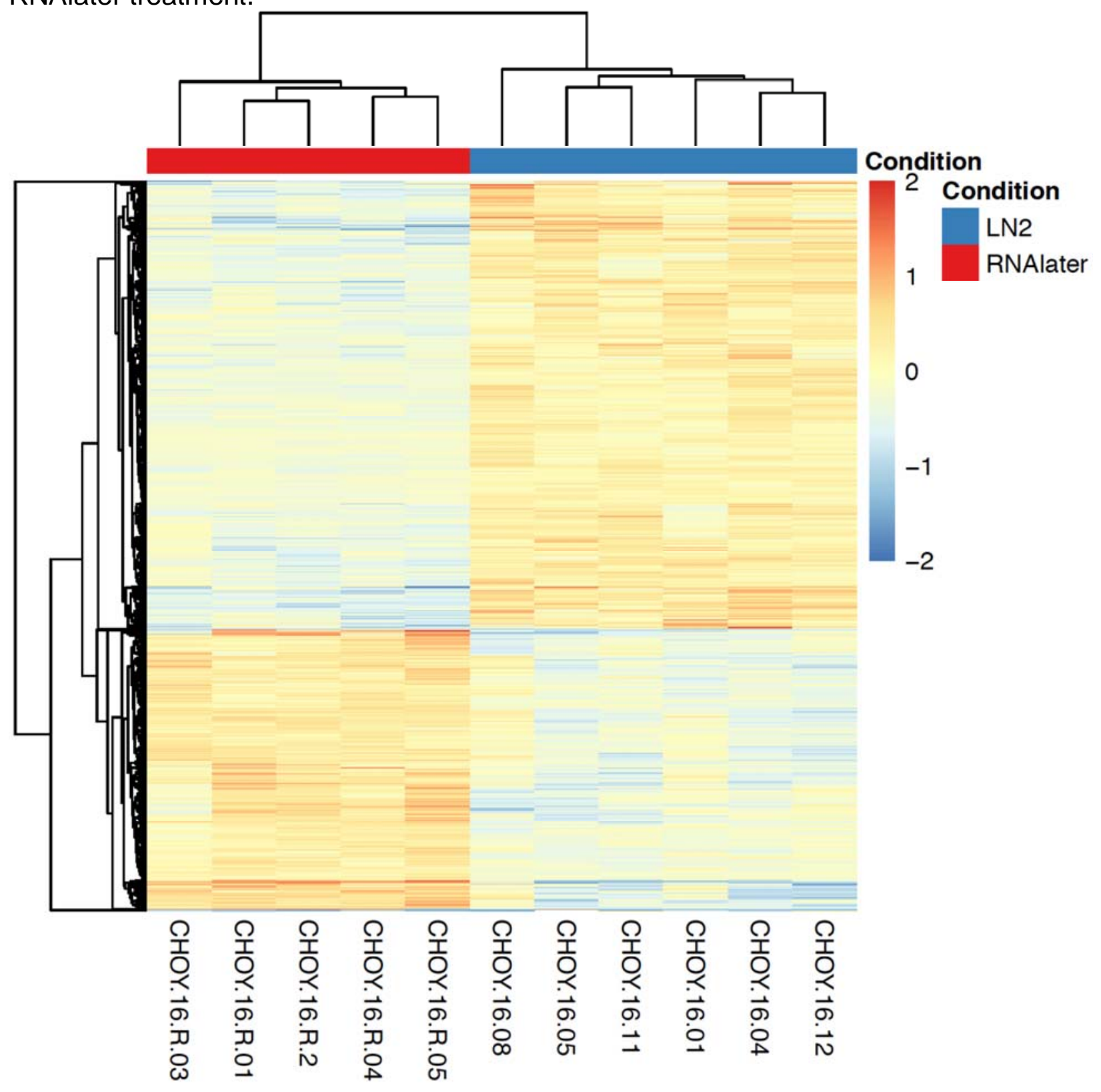


532 Figure 3: Relationships among the dependent variables retained in the best-fitting 533 generalized linear model. M: mean expression; G: GC content; E: exon number; H: 534 homopolymer repeat presence/absence.
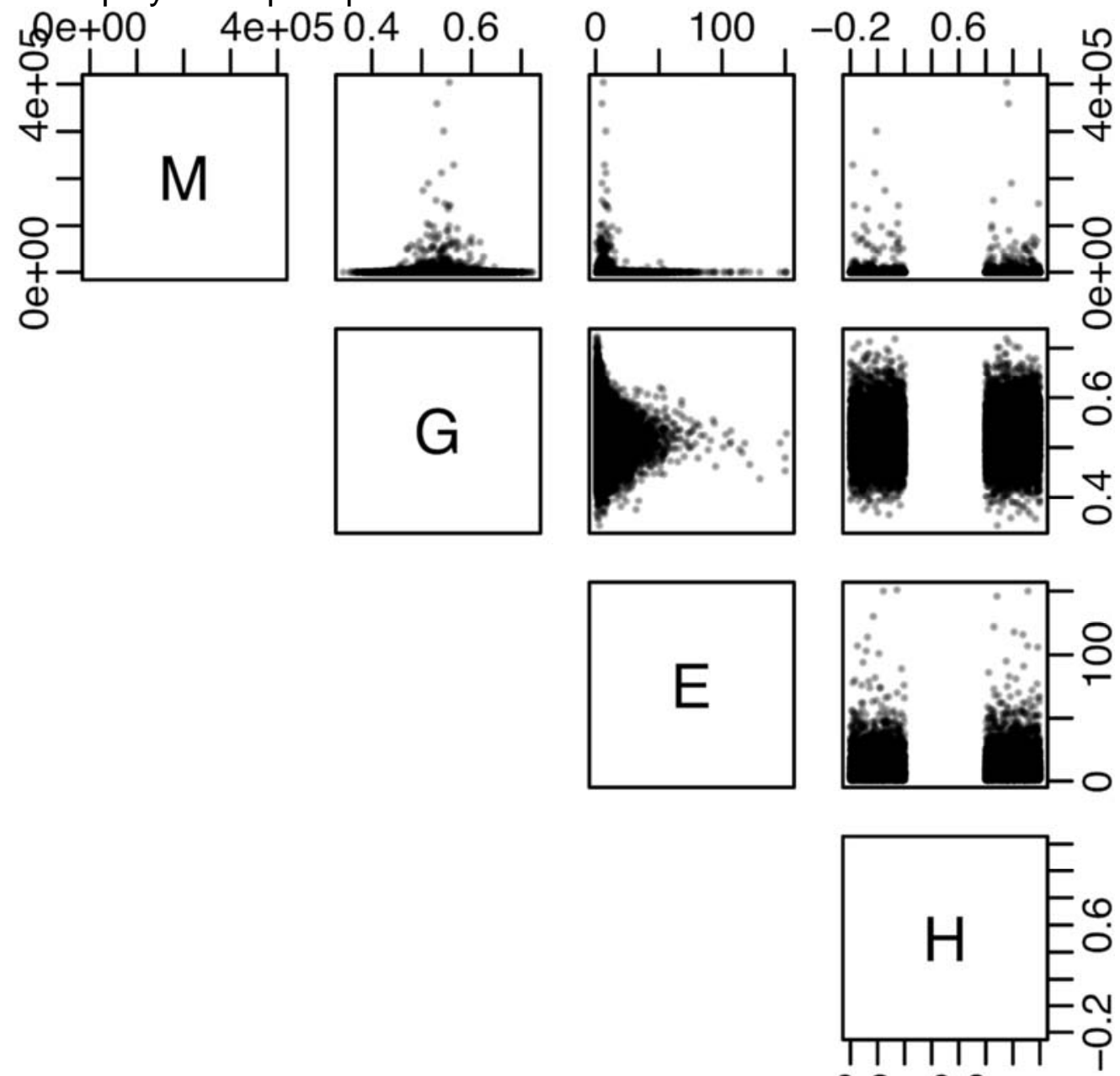
536 Supplementary material

537 Table S1: All samples were collected at January 7, 2017 at 10pm EST and were exactly 30-day old fry from the same

538 clutch. RNAlater samples were left on the bench top for 17 days prior to extraction. Liquid N2 samples were flash frozen

539 and stored at $-80^{\square} \mathrm{C}$ prior to extraction. Reported are the treatments (RNALater vs. liquid nitrogen), sample name,

540 extraction date, extraction time, concentration ( $\mathrm{ng} / \mathrm{uL}$ ) based on ribogreen, lane the sample was sequenced in and RNA

541 integrity (RIN) scores calculated using RNA bioanalyzer.

542

543

544

545

\section{Treatment Sample}

RNAlater

RNAlater

546 RNAlater

547 RNAlater

548 RNAlater

549 LiquidN2

550 LiquidN2

551 LiquidN2

552 LiquidN2

553 LiquidN2

554 LiquidN2

$\begin{array}{ll}\text { Sample } & \text { extract_date } \\ \text { CHOY-16-R-1 } & 1 / 24 / 17 \\ \text { CHOY-16-R-2 } & 1 / 24 / 17 \\ \text { CHOY-16-R-3 } & 1 / 24 / 17 \\ \text { CHOY-16-R-4 } & 1 / 24 / 17 \\ \text { CHOY-16-R-5 } & 1 / 24 / 17 \\ \text { CHOY-16-01 } & 1 / 23 / 17 \\ \text { CHOY-16-04 } & 1 / 24 / 17 \\ \text { CHOY-16-05 } & 1 / 22 / 17 \\ \text { CHOY-16-08 } & 1 / 21 / 17 \\ \text { CHOY-16-11 } & 1 / 19 / 17 \\ \text { CHOY-16-12 } & 1 / 19 / 17\end{array}$

extract_time

5:30 PM

5:30 PM

5:30 PM

5:30 PM

5:30 PM

3:30 PM

1:30 PM

3:30 PM

3:30 PM

12:00 PM

6:00 PM

$\begin{array}{lll}\text { ng/uL } & \text { Lane } & \text { RIN } \\ 287.53 & 7 & 8.3 \\ 83.94 & 4 & 8.4 \\ 39.30 & 8 & 8.8 \\ 38.71 & 1 & 8.7 \\ 52.54 & 3 & 8.8 \\ 264 & 6 & 10.0 \\ 144.76 & 5 & 9.9 \\ 69.10 & 8 & 10.0 \\ 102.10 & 2 & 9.5 \\ 78.88 & 6 & 10.0 \\ 67.32 & 5 & 9.6\end{array}$

555 
556 Figure S1: Boxplots depicting normalized counts from DESeq2 for RNALater and liquid 557 nitrogen stored samples. Counts were log transformed (log(1+counts)) for all libraries.

558 A) shows raw counts, and B) shows counts that were filtered for genes with $\leq 100$ counts 559 across all samples.

560
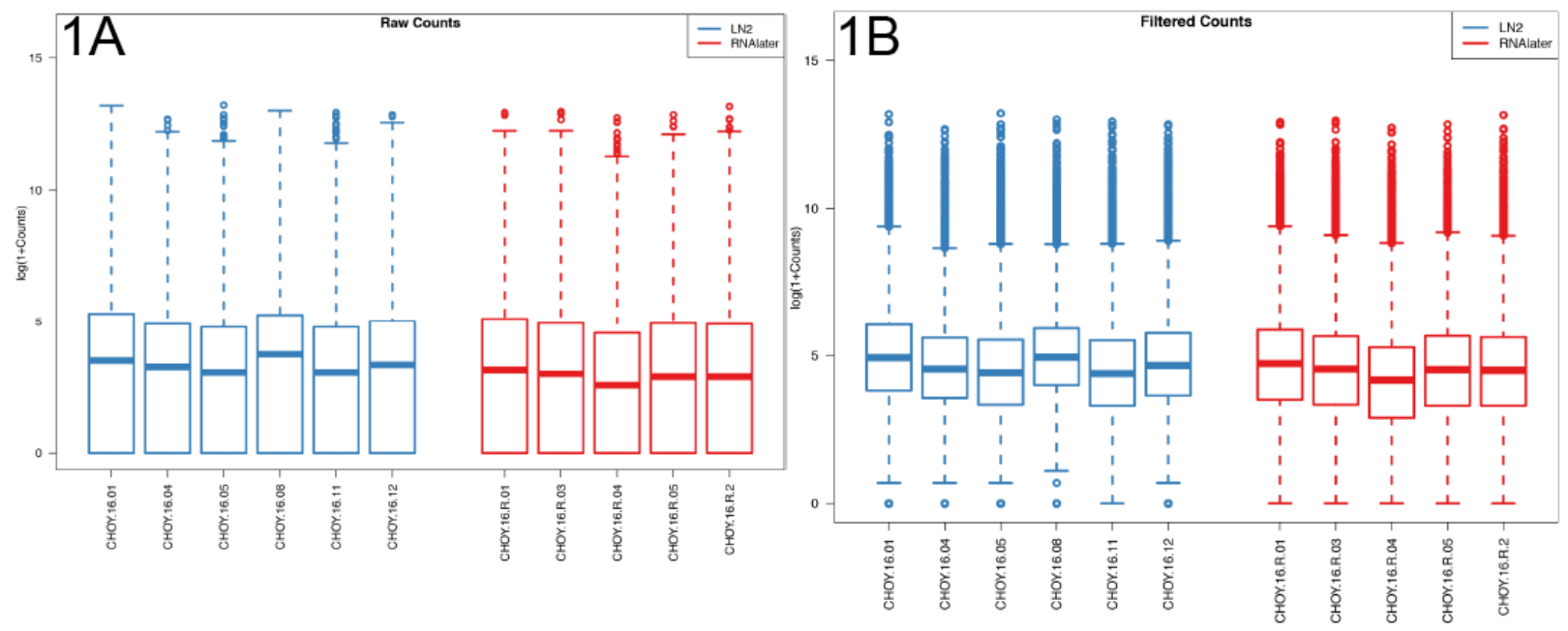
564 Figure S2: Scatterplot showing the relationships and distributions of all predictors 565 tested in the linear model. $M$ is the mean expression across all samples, $L$ is the 566 annotated gene length, $G$ is the $G C$ content, $E$ is the number of annotated exons, $S$ is 567 simple sequence repeat presence, and $\mathrm{H}$ is homopolymer repeat presence. $\mathrm{S}$ and $\mathrm{H}$ 568 have been jittered to avoid overplotting. Each gene is represented by one point in each 569 scatterplot cell.
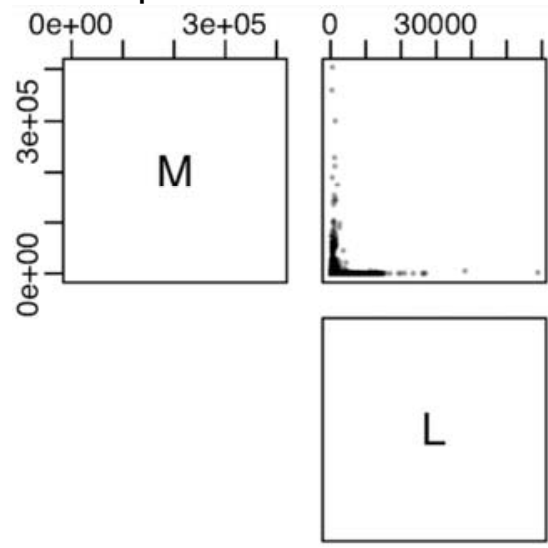
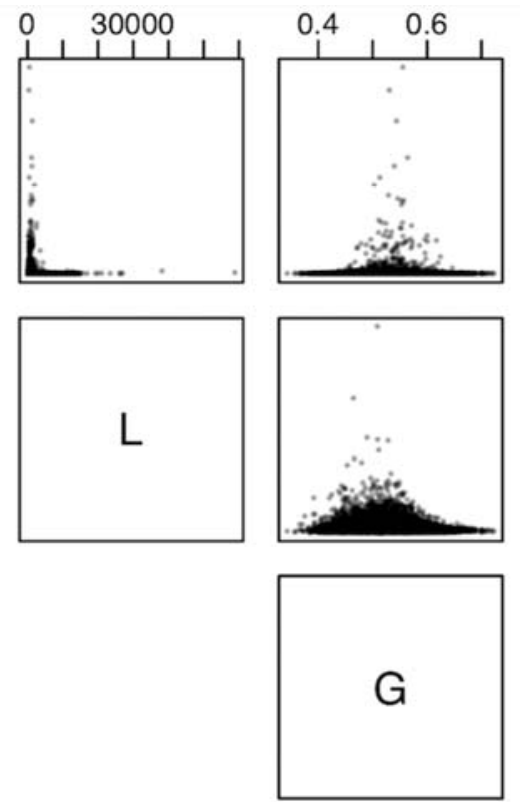

$\mathrm{G}$

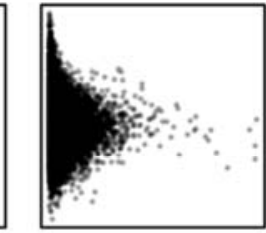

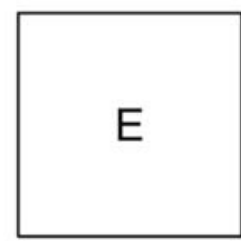
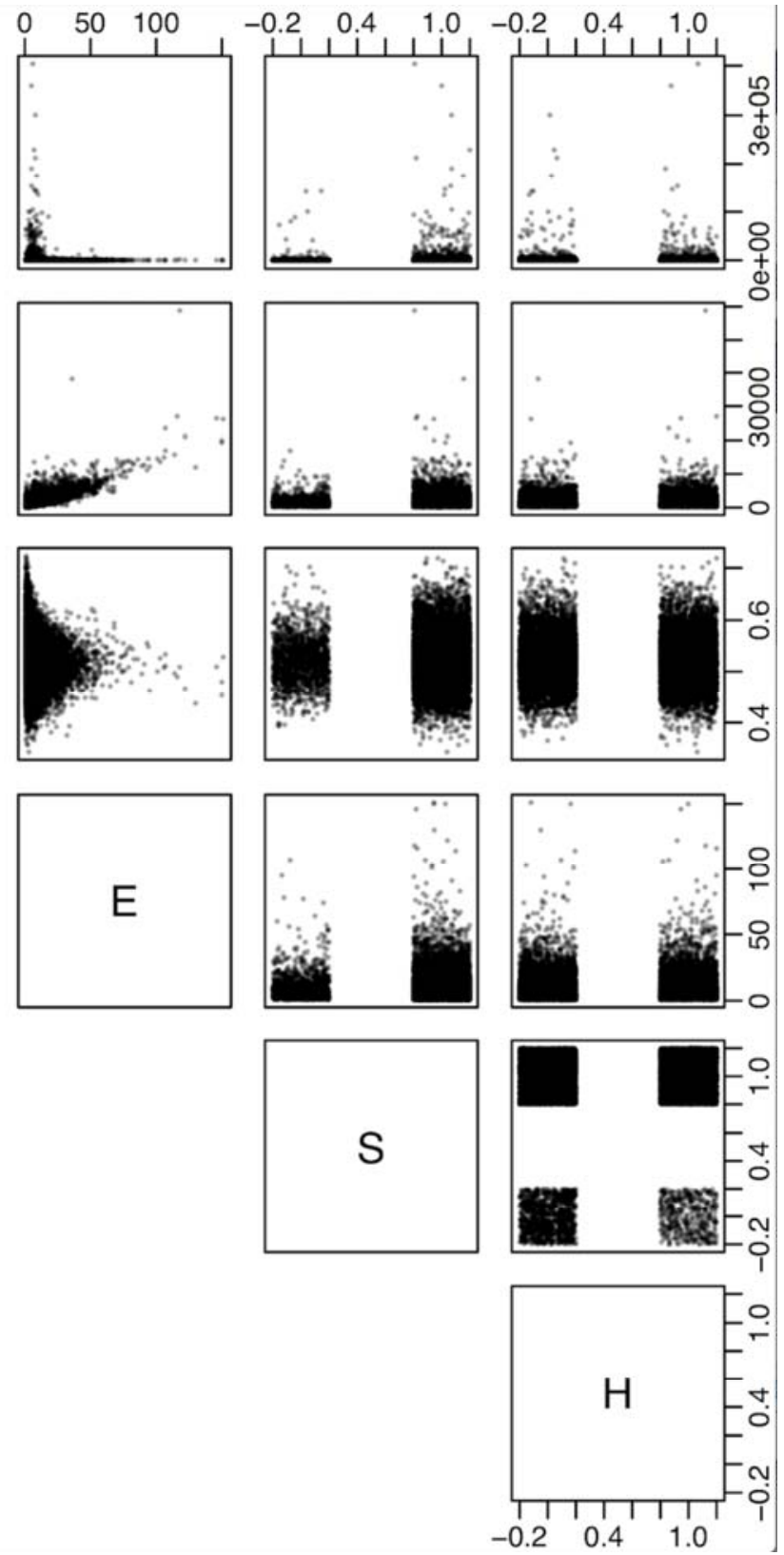
573 Figure S3: Box plots depicting GC content of genes that were differentially expressed 574 between treatments (e.g. "Higher exp. in") and all genes that passed the filtering 575 thresholds (e.g. "All Genes").
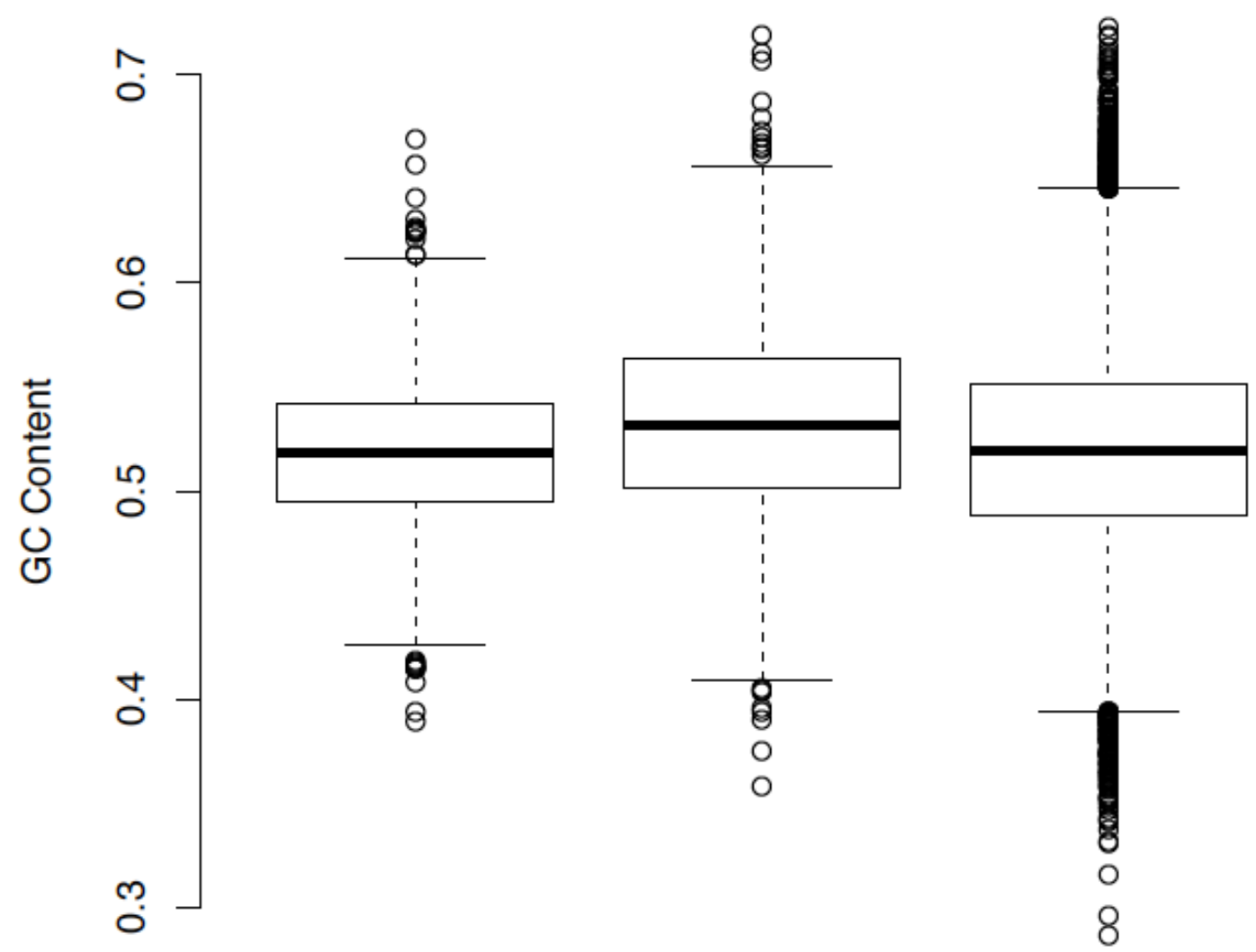

Higher exp. in RNAlater

Higher exp. in LN2

All Genes 


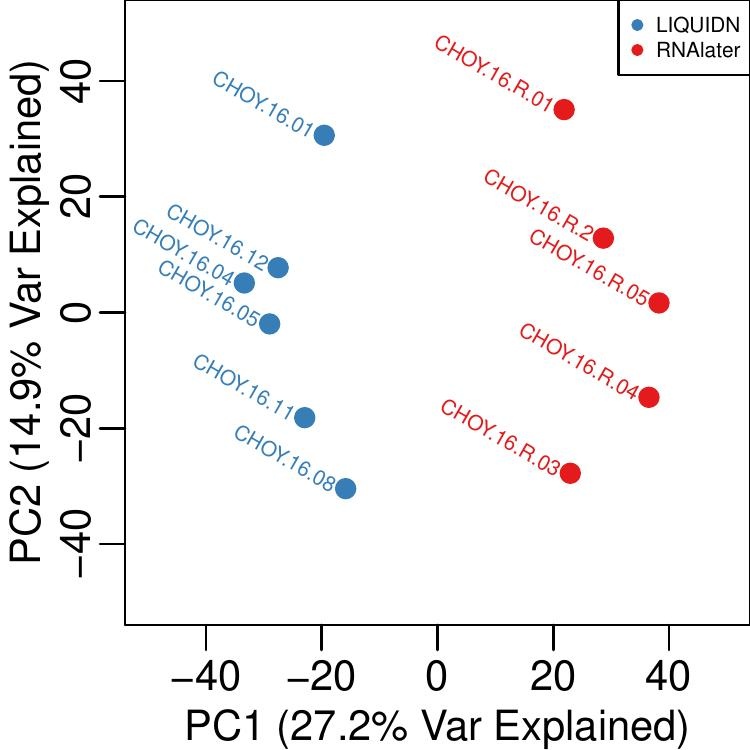




\section{Condition}

2 Condition
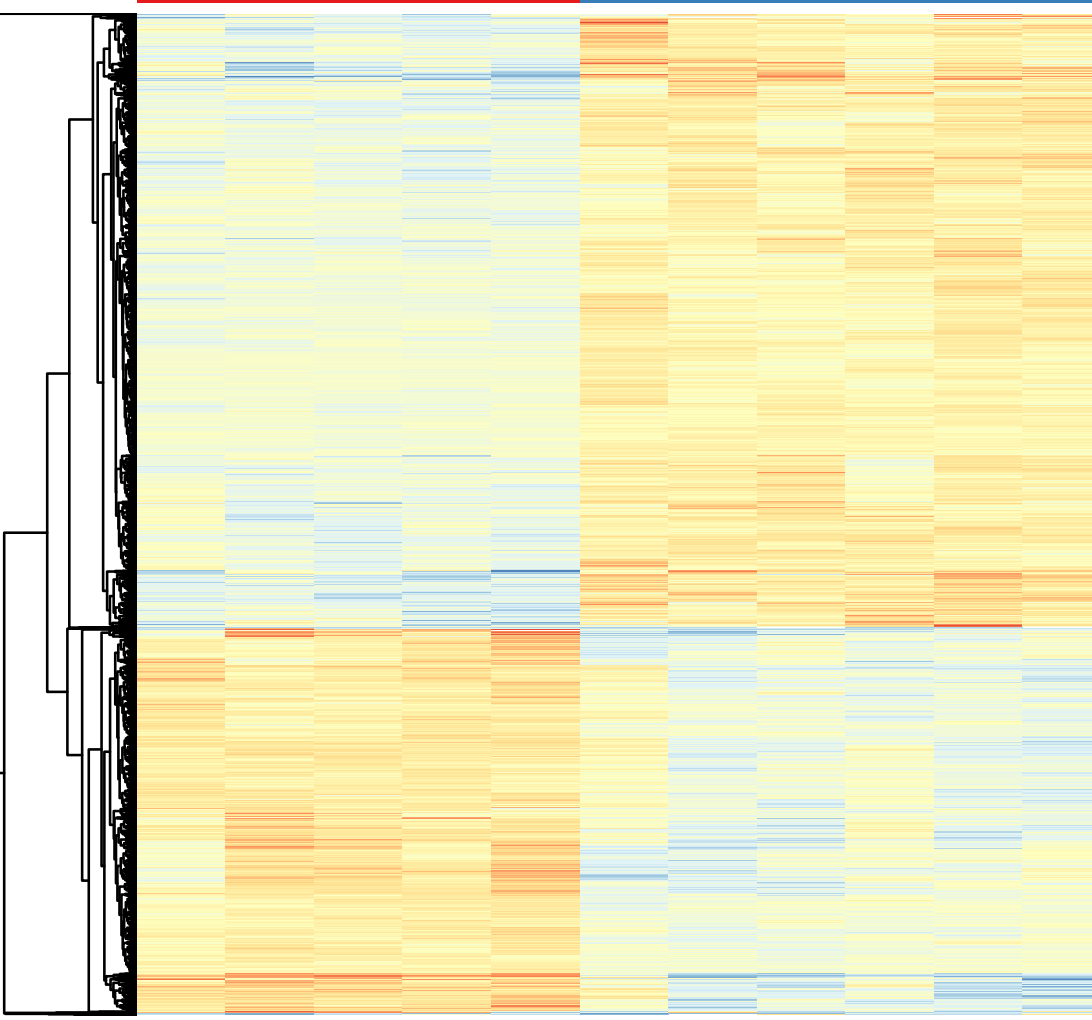


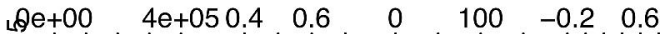
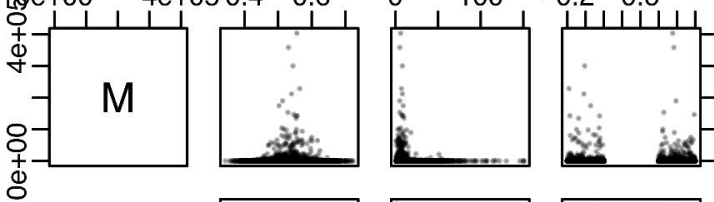

ما
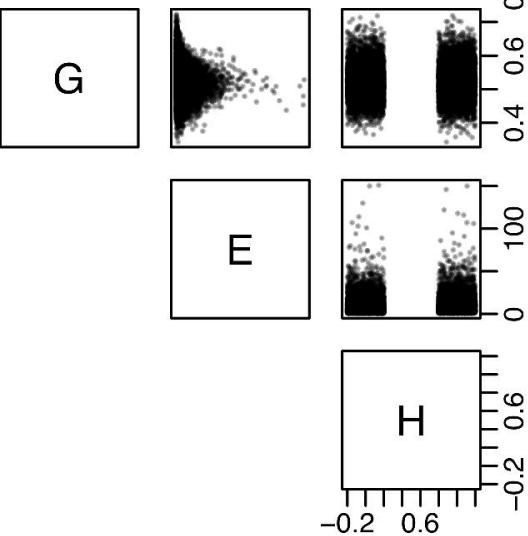


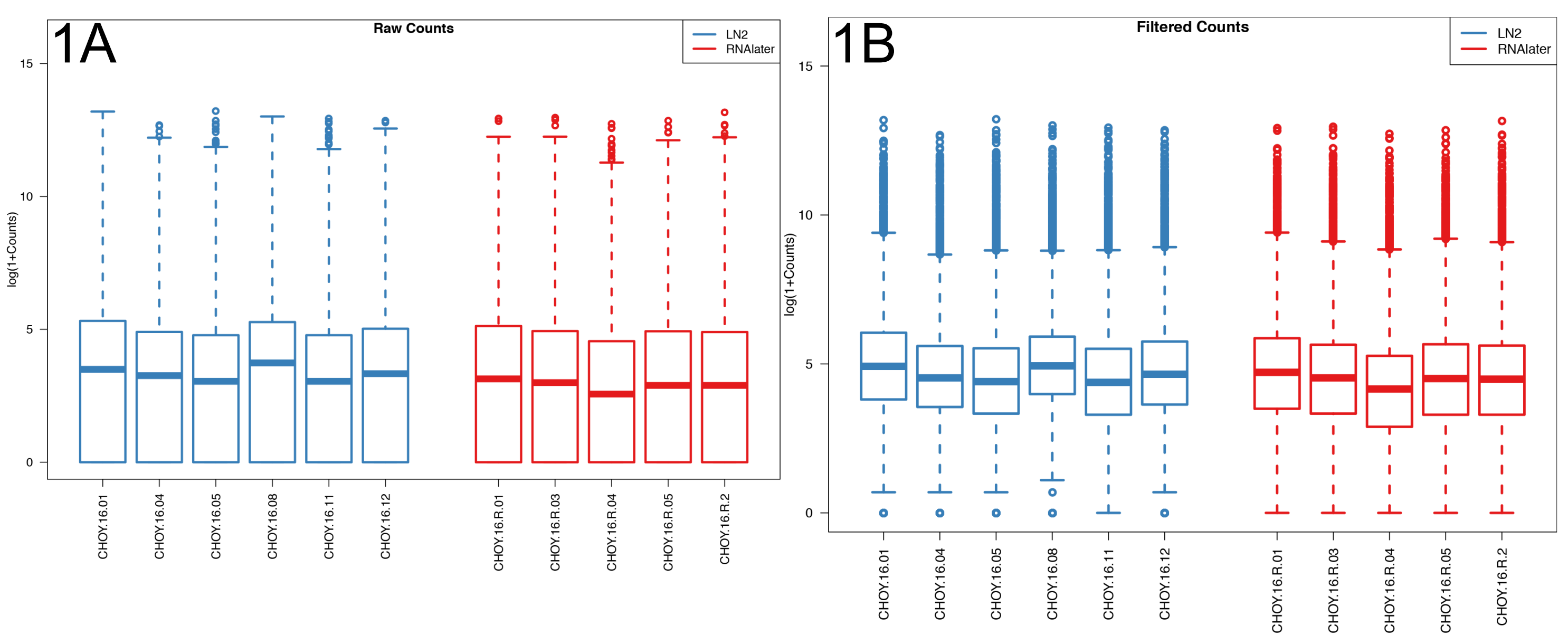




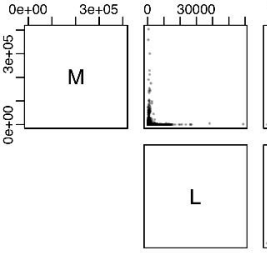

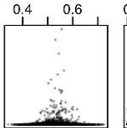
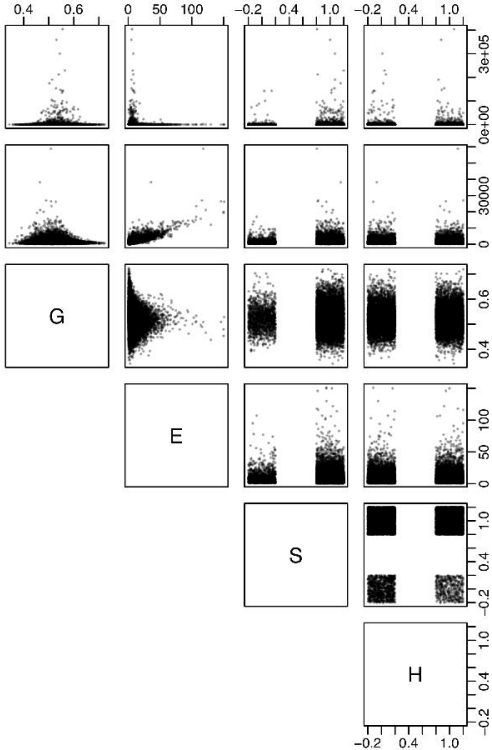

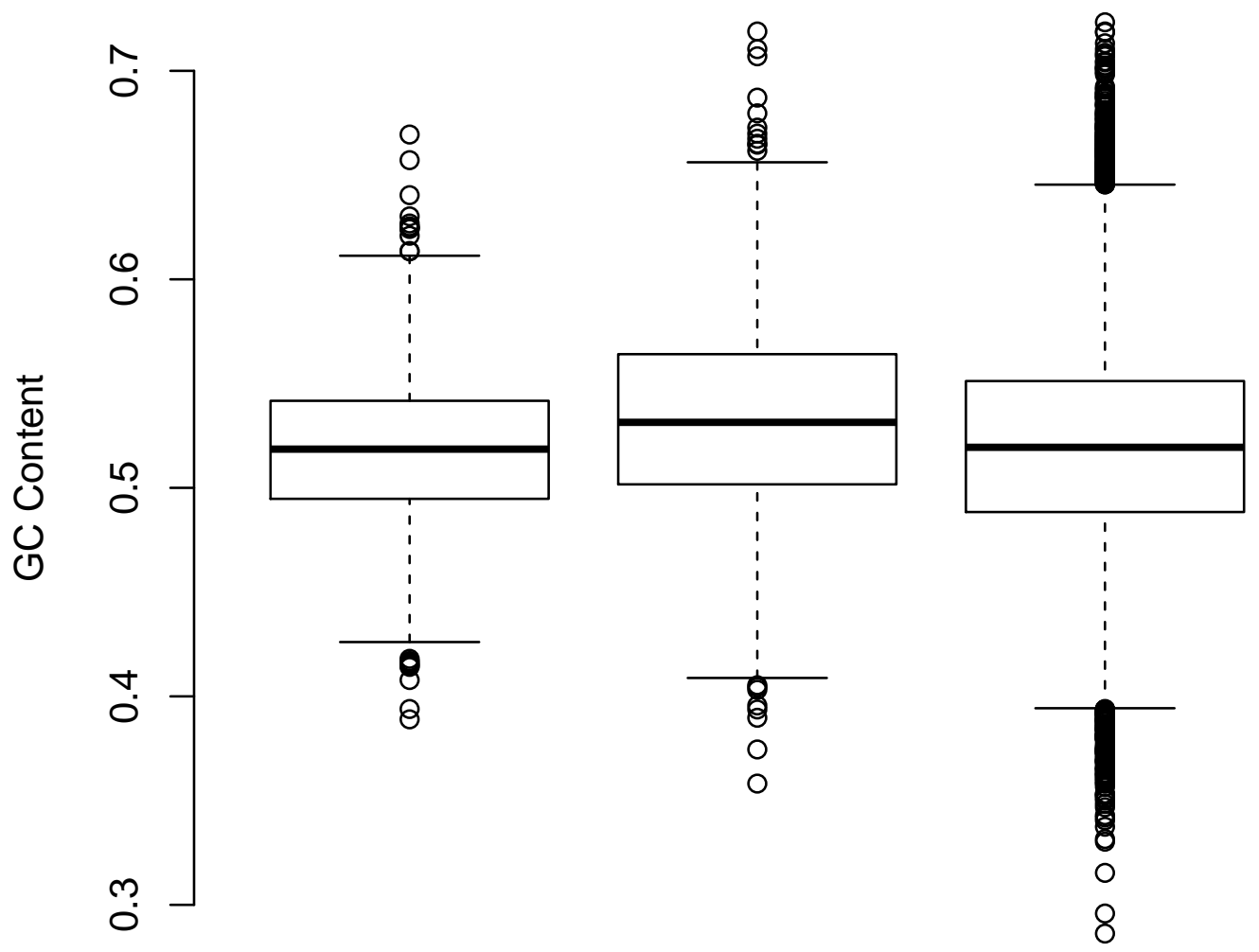

Higher exp. in RNAlater

Higher exp.

in LN2

All Genes

Category 